\title{
Nonlinear properties of high-Q optical microresonators in normal dispersion range
}

\author{
Artem Shitikov ${ }^{1,2, *}$, Valery Lobanov ${ }^{1}$, Nikolay Pavlov ${ }^{1,3}$, Andrey Voloshin ${ }^{1}$, Igor Bilenko ${ }^{1,2}$, \\ and Michael Gorodetsky ${ }^{1,2}$ \\ ${ }^{1}$ Russian Quantum Center, Moscow, Russia \\ ${ }^{2}$ Lomonosov Moscow State University, Faculty of Physics, Moscow, Russia \\ ${ }^{3}$ Moscow Institute of Physics and Technology, Dolgoprudny, Russia
}

\begin{abstract}
We demonstrate the generation of Kerr frequency combs and platicons in whispering gallery mode crystalline microresonators in normal group velocity regime at $780 \mathrm{~nm}$ and $1064 \mathrm{~nm}$ wavelengths.
\end{abstract}

Whispering gallery mode (WGM) microresonators can have extremely high values of quality-factor (exceeding 109) in the optical range, that allows low threshold of many interesting nonlinear effects, in particular, the generation of Kerr frequency combs [1]. Kerr combs in optical microresonators may become a basis for a new class of compact devices for spectroscopy, precision measurements, and generation of femtosecond pulses.

Coherent low-noise frequency combs are of particular interest. These combs may be generated in the form of dissipative Kerr solitons in the region of anomalous group velocity dispersion (GVD) [2] and to date have been already demonstrated on different platforms. However, most materials suitable for the manufacturing of WGM microresonators (for example, widely used fluorides $\mathrm{MgF}_{2}$ and $\mathrm{CaF}_{2}$ ) have a normal GVD in the visible and near IR ranges, which prevents soft generation of coherent frequency combs and bright dissipative Kerr solitons. Therefore, the development of new methods for coherent frequency comb excitation in microresonators with normal GVD and studying the properties of such combs is an actual and practically significant task. It was shown recently that in the normal GVD regime coherent frequency combs could be achieved in the form of soliton-like pulses of a particular form, so-called platicons [3]. It was also demonstrated that the efficiency of the transformation of pump power into the power of the frequency comb lines is significantly better for platicons compared to the generation of bright solitons in anomalous GVD regime. This properties of platicons are important for many practical applications.

In the recent years, several methods for the generation of platicons were proposed: the use of a local dispersion perturbations caused by mode interactions $[3,4]$, pump modulation at a frequency equal to the free spectral range (FSR) of a microresonator and biharmonic pump [5], self-injection locking of a laser source to a mode of the WGM microresonator [6].

In our work $\mathrm{MgF}_{2}$ microresonators and prism coupling were used. For $3 \mathrm{~mm}$ in diameter $\mathrm{MgF}_{2}$ microresonators the GVD is mostly determined by material dispersion which is normal at given wavelengths. The microresonator's loaded quality-factor exceeded $5 \times 10^{8}$.

For the pump at $1064 \mathrm{~nm}$, the experimental setup was adapted both for biharmonic and for the amplitude-modulated pump (see Fig. 1). For the pump at $780 \mathrm{~nm}$, a setup with an

\footnotetext{
*Corresponding author: Shartev@gmail.com
} 
external cavity diode laser self-injection-locked to a mode of the same high-Q WGM microcavity was used (Fig. 1, on right). The experimental results showed that, due to the effect of self-injection locking, the generated linewidth may become less than $1 \mathrm{kHz}$.
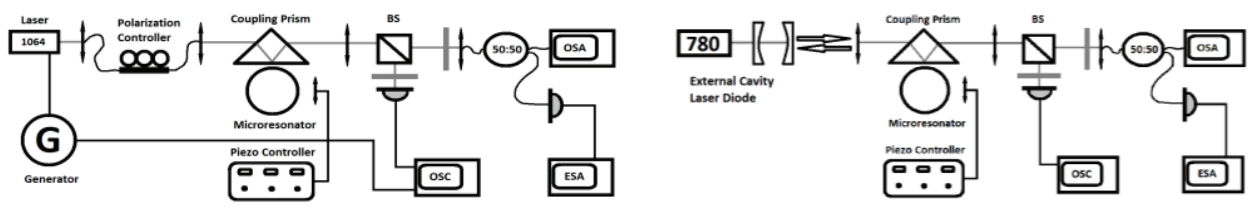

Fig. 1. Experimental setup for the pump at $1064 \mathrm{~nm}$; self-injection locked pump at $780 \mathrm{~nm}$ (right).

For the pump at $1064 \mathrm{~nm}$, combs were observed for all modes with a quality-factor exceeding $5 \times 10^{8}$ and coupling efficiency better than $10 \%$. Frequency combs with asymmetric spectral envelope caused by mode interaction were observed. High-efficiency power conversion into comb lines (more than 30\%) was also observed.

For the pump at $780 \mathrm{~nm}$, in addition to the combs similar to the combs obtained at $1064 \mathrm{~nm}$, platicon-like spectra with a width of 1-3 nm were also regularly detected. The characteristic plateau of the spectrum was $20 \mathrm{dBm}$ below the pump line. The width of the microwave beatnote signal between the comb lines was less than $1 \mathrm{kHz}$ and was detected on an electrical spectrum analyzer at a frequency corresponding to the FSR of microresonator.

The results of the experiments are shown in Fig. 2.
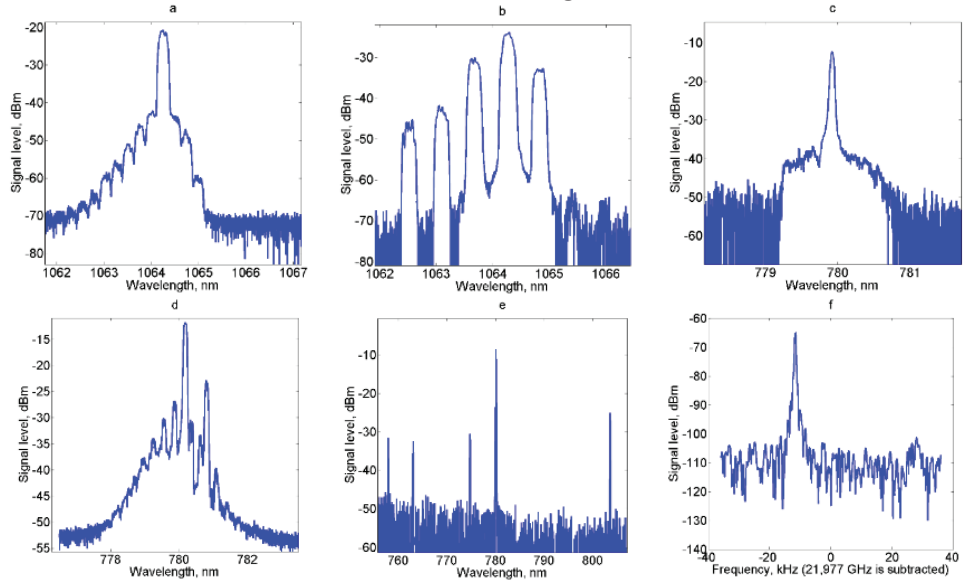

Fig. 2. a, b - frequency combs, formed as a result of mode interaction with pump at $1064 \mathrm{~nm}$ : a - the distance between the lines corresponds to 3 FSR; $b$ - the first lines are below the pump by $6 \mathrm{~dB}$, the generation efficiency is more than $30 \%$. c, d, e - combs generated by the self-injection-locked diode laser pumping at $780 \mathrm{~nm} . \mathrm{c}-$ is a platicon-like spectrum with a width of $1.5 \mathrm{~nm} . \mathrm{d}$ - platicon-like spectrum with pronounced comb lines. e - hyperparametric generation in the range of $50 \mathrm{~nm}$. $\mathrm{f}-$ the beatnote between the comb lines, the line width is less than $1 \mathrm{kHz}$.

Experimental setups for the of optical frequency combs generation in WGM microresonators with normal GVD were designed and tested. It is planned to investigate the combs properties and to compare different methods of its excitation.

This work was supported by the Russian Science Foundation (project №17-12-01413).

\section{References}

[1] T. Herr, K. Hartinger et al., Nature Photon. 6, 48 (2012)

[2] V.E. Lobanov, G. Lihachev et al., Opt. Exp. 24(24), 27382 (2016)

[3] V.E. Lobanov, G. Lihachev et al., Opt. Exp. 23(6) 7713 (2015)

[4] X.X. Xue, Y. Xuan et al., Laser Photon. Rev. 9, L23 (2015)

[5] V.E. Lobanov, G. Lihachev, M. Gorodetsky, Europhys. Lett. 112, 5 (2015)

[6] W. Liang, A.A. Savchenkov et al., Opt. Lett. 39, 2920 (2014) 